predictors of death identified female sex, mild Charlson Comorbidity Index (chronic illness without cancer), and Northeast hospital location. Mortality tripled in those requiring mechanical ventilation $(7.43 \%$ vs $2.22 \% ; \mathrm{p}<0.0001)$, mostly older patients. Potential etiologies and complications of GCSE that predicted mortality included cerebrovascular disease, hypoxic-ischemic brain injury, cardiac disease, respiratory failure, and higher comorbidity index. In the first decade of life, GCSE is the most common neurologic emergency. The number of cases $<10$ years old in this cohort $(2,524,21.8 \%)$ was comparable to all cases older than 60 years of age $(2,627,22.7 \%)$. (Koubeissi M, Alshekhlee A. Inhospital mortality of generalized convulsive status epilepticus: a large US sample. Neurology August 28, 2007;69:886-893). (Reprints: Dr Mohamad Z Koubeissi, Department of Neurology, University Hospitals Case Medical Center, Case Western Reserve University, 11100 Euclid Ave, Cleveland, OH 44106).

COMMENT. A bimodal age distribution of cases of GCSE, with highest frequencies in children $<10$ years and in the elderly is similar to other reports. The low mortality rate in young children in this study, lower than some previous series, is explained by the authors as a reflection of improved management and availability of rectal antiepileptic medications, or the relatively low frequency of acute symptomatic epilepsies in their cohort. A more detailed analysis of etiological factors related to GCSE outcome in children would be of interest, especially in children $<1-2$ years of age, when the incidence is highest and febrile CSE is the most common cause.

\title{
EPIDEMIOLOGY OF STATUS EPILEPTICUS IN CHILDREN
}

The incidence, etiology, seizure characteristics, and outcome in childhood convulsive status epilepticus (CSE) are reviewed by researchers from Great Ormond Street Hospital for Children, and the Institute of Child Health, London, UK. Neonatal seizures require separate consideration and were excluded. Despite attempts to modify the definition of CSE, without a set temporal criterion (ILAE (2001), or a duration as low as 5 min (operational definition), there is no clear reason to modify the "traditional" 30-min-duration definition. By 30 min CSE has become self-sustaining, drug resistance has occurred, and neuronal injury has begun.

Etiology is an important determinant of CSE, and a significant proportion of cases are associated with fever. ILAE classification of SE according to etiologies (1993) lists 1) acute symptomatic - previously neurologically normal child, within 1 week of underlying etiology including CNS infection, prolonged febrile seizure, encephalopathy, head trauma, cerebrovascular disease, metabolic or toxic; 2) remote symptomatic - preexisting CNS disorder $>1$ week before; 3) idiopathic epilepsy related; 4) cryptogenic epilepsy related; and 5) Unclassified. This classification should be revised, with febrile CSE as a distinct category with an overall favorable prognosis, and separate from acute symptomatic CSE.

Incidence estimates of CSE in children are few, the most recent conducted in North London, the only study of a wholly pediatric population. The incidence of childhood CSE in North London is 18-20/100,000/year, higher than the 4-6/100,000/year reported in adults, excluding the elderly. The incidence in children less than 1 year is $51 / 100,000 /$ year, compared to those aged 1-4 (29/100,000/year). The higher frequency of CSE in the very young is related to a high proportion of acute symptomatic causes, to brain immaturity, or 
congenital, genetic, and metabolic disorders in this age group. Future studies should clarify the role of CSE in mesial temporal sclerosis and seizures, and identify specific ethnic, genetic, or socioeconomic factors in etiology and methods of CSE prevention. (RaspallChaure M, Chin RFM, Neville BG, Bedford H, Scott RC. The epidemiology of convulsive status epilepticus in children: a critical review. Epilepsia Sept 2007;48:1652-1663). (Reprints: Dr Rod C Scott, Senior Lecturer in Paediatric Neurosciences, Paediatric Neurologist, Institute of Child Health, The Wolfson Centre, Mecklenburgh Square, London WC1 N2AP, UK).

COMMENT. In a current Dutch study of SE in children with epilepsy, $41(8.3 \%)$ of 494 had one or more episodes of SE at the time of epilepsy diagnosis. Three had febrile SE, followed by unprovoked seizures, and 38 were unprovoked SE. (Stroink H, et al. Epilepsia Sept 2007;48:1708-1715). SE was the first seizure in 32 (78\%). After 5 year follow-up, $31.7 \%$ of patients with $\mathrm{SE}$ at onset had a shorter terminal remission $<1$ year compared to $21.2 \%$ of those without SE. Mortality was not significantly increased for children with SE. Risk factors for SE at onset of epilepsy were remote symptomatic and cryptogenic etiology, and a history of febrile seizures. SE during the course of the epilepsy has a worse prognosis and a high recurrence rate of SE.

\section{SIMPLE FEBRILE SEIZURES AND TEMPORAL LOBE EPILEPSY ASSOCIATED WITH SCN1A MUTATION}

The electroclinical features of a large Italian family with 13 living members having simple febrile seizures and SCN1A missense mutation (M145T), some complicated by temporal lobe epilepsy, are reported from University Magna Graecia, Catanzaro, and other centers in Italy. All 13 affected members ( 7 male, mean age $36.6+/-20.4$ years) had febrile seizures up to the age of 6 years. Age at onset of febrile seizures ranged from 5 to 45 months, mean age of $12.8+/-12.9$ months. EEGs were normal in 9 with simple febrile seizures only. Three developed temporal lobe epilepsy, 2 having MRI evidence of mesial sclerosis. (Colosimo E, Gambardella A, Mantegazza M, et al. Electroclinical features of a family with simple febrile seizures and temporal lobe epilepsy associated with SCN1A loss-of-function mutation. Epilepsia Sept 2007;48:1691-1696). (Reprints: Prof Antonio Gambardella, Cattedra ed UO di Neurologia, Universita degli Studi Magna Graecia, Campus Universitario di Germaneto, Viale Europa, 88100 Catanzaro, Italy).E-mail: a.gambardella@isn.cnr.it

COMMENT. SCN1A mutations may cause simple febrile seizures later associated with temporal lobe epilepsy, and separate from the characteristic association with generalized epilepsy with febrile seizures plus (GEFS+).

A frequency of $71 \%$ SCN1A abnormalities are associated with severe myoclonic epilepsy of infancy (SMEI) including borderline SMEI (SMEB), and $11.5 \%$ with GEFS+ probands. (Marini $\mathrm{C}$ et al. Idiopathic epilepsies with seizures precipitated by fever and SCN1A abnormalities. Epilepsia Sept 2005;48:1678-1685). 